Supporting Information

\title{
Rapid Ion Transport Induced by the Enhanced Interaction in Composite Polymer Electrolyte for All-solid-state Lithium-metal Batteries
}

Yanan Xu ${ }^{a, b}$, Kai Wang a,b,c*, Yabin An ${ }^{a, b}$, Wenjie Liu ${ }^{a, b}$, Chen Li ${ }^{a, b}$, Shuanghao

Zheng, ${ }^{c, d}$ Xiong Zhang ${ }^{a, b, c}$, Lei Wang ${ }^{a, b}$, Xianzhong Sun ${ }^{a, b}$, Yanwei Ma ${ }^{a, b^{*}}$

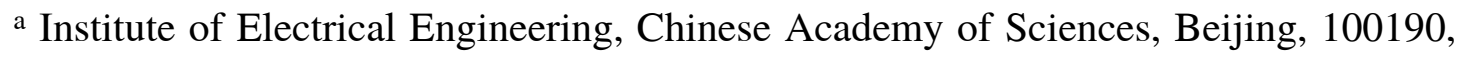
China

b School of Engineering Sciences, University of Chinese Academy of Sciences, Beijing, 100049, China

${ }^{\mathrm{c}}$ Dalian National Laboratory for Clean Energy, Chinese Academy of Sciences, Dalian 116023, China

d State Key Laboratory of Catalysis, Dalian Institute of Chemical Physics, Chinese Academy of Sciences, 457 Zhongshan Road, Dalian 116023 China

AUTHOR INFORMATION

Corresponding Author

* Kai Wang, *Yanwei Ma 
Email:wangkai@mail.iee.ac.cn; ywma@mail.iee.ac.cn

\section{Experimental}

\section{Materials preparation}

PVDF-HFP (Mw: 300,000) and LiTFSI was purchased from Sigma-Aldrich and dried under vacuum at $100^{\circ} \mathrm{C}$ for $24 \mathrm{~h}$ before use. The garnet M-LLZTO was synthesized by solid-state reaction according to reference. ${ }^{28} \mathrm{~N}-\mathrm{LLZTO}$ particles were obtained by ball milling of M-LLZTO for $48 \mathrm{~h}$. The cathode material $\mathrm{LiFePO}_{4}$ was purchased from BTR New Material Group Co., Ltd.

The composite solid electrolyte membranes were prepared by a simple film casting method. Typically, PVDF-HFP was first dissolved in DMF solvent and stirred for several hours until it was completely dissolved. Then, LiTFSI salts were added and stirred to obtain a homogeneous solution. The mass ratio of PVDF-HFP and LiTFSI was 5:3. Next, the N-LLZTO particles were added into the above solution in which the content of N-LLZTO was $0,10,20$, and $30 \mathrm{wt} \%$ of the total mass of PVDF, LiTFSI and N-LLZTO. The resulting suspension was then stirred for another several hours to ensure that the N-LLZTO is evenly dispersed. After that, the mixture was cast on a clean glass plate and automatically spread under gravity. Finally, the composite solid polymer electrolyte (CPE) membranes with average thickness of $\sim 50$ $\mu \mathrm{m}$ could be achieved by drying at $120^{\circ} \mathrm{C}$ for $24 \mathrm{~h}$ in vacuum oven to remove the DMF solvent. The obtained CPEs were denoted as CPE-0, CPE-10, CPE-20, and CPE-30, respectively. 


\section{Materials characterization}

$\mathrm{X}$-ray diffraction (XRD) measurements were carried out on a D8 Advance X-ray diffractometer with a nonmonochromated $\mathrm{Cu} \mathrm{K \alpha}$ X-ray source to characterize the crystalline structure of materials and obtained CPEs. Scanning electron microscope (SEM) images were performed with a Hitachi S-4800 microscope at an acceleration voltage of $20 \mathrm{kV}$. Transmission electron microscopy (TEM) images were recorded by using a JEOL JEM-2100F. Electron probe micro-analyzer (EPMA) element mappings were collected on a JEOL JXA8320. Fourier transform infrared (FTIR) spectral measurements were used on a Cary 630 FTIR. TGA curves were conducted on a Netzsch STA $449 \mathrm{~F} 5$ under a heating rate of $10^{\circ} \mathrm{C} \mathrm{min}^{-1}$ in air atmosphere. Solid-state ${ }^{7} \mathrm{Li}$ NMR spectra were performed on a Bruker Avance III-400.

\section{Electrochemical characterization of the CPEs}

Electrochemical impedance spectroscopy (EIS) was tested at the temperature range from 30 to $80^{\circ} \mathrm{C}$ and a frequency range between $100 \mathrm{kHz}$ and $1 \mathrm{~Hz}$ by a VMP3 electrochemical station (Bio-Logic Co., France) to calculate the ionic conductivities of CPEs. The CPE membrane was sandwiched between two stainless steel (SS) electrodes to determine the ionic conductivity. The ionic conductivity $(\sigma)$ was calculated by the equation (S1):

$$
\sigma=\frac{L}{R S}
$$

where $L$ is the thickness of electrolyte membrane, $S$ is the effective contact area, and 
$R$ is the bulk resistance. Linear sweep voltammetry (LSV) was measured to evaluate the electrochemical window of CPEs by assembling SS|CPE|Li coin cells from 2 to 6 V. The Li transference number $\left(t_{+}\right)$was measured by alternating current impedance and direct current polarization in a $\mathrm{Li}|\mathrm{CPE}| \mathrm{Li}$ symmetric cell, and calculated by the equation (S2):

$t_{+}=\frac{I_{s s}\left(\Delta \mathrm{V}-I_{0} R_{0}\right)}{I_{0}\left(\Delta \mathrm{V}-I_{s s} R_{s s}\right)}$

where $I_{0}$ and $I_{S S}$ are the initial and steady-state currents, $\Delta V$ is the polarization voltage (10 $\mathrm{mV})$, and $R_{0}$ and $R_{s s}$ are the initial and steady-state interfacial resistance, respectively. The galvanostatic cycling test was carried out with symmetric $\mathrm{Li}|\mathrm{CPE}| \mathrm{Li}$ cells using a Neware CT-4008 battery tester at room temperature with charging for 1 $\mathrm{h}$ and discharging for $1 \mathrm{~h}$ at different current densities.

\section{Electrochemical characterization of $\mathrm{LiFePO}_{4}|\mathrm{CPE}| \mathrm{Li}$ Cells}

The cathode was prepared by mixing $\mathrm{LiFePO}_{4}$, carbon black (Super-P), and PVDF (80:15:5 by weight) in N-methylpyrrolidinone (NMP) solvent to form a homogeneous slurry. Then the slurry was cast onto a carbon-coated aluminum foil and dried in a vacuum oven at $100{ }^{\circ} \mathrm{C}$ overnight. The active material loading of was about $3.5 \mathrm{mg} \mathrm{cm}^{-2}$. The thickness of the lithium foil was $20 \mu \mathrm{m}$. For CR2032-type $\mathrm{LiFePO}_{4}|\mathrm{CPE}-20| \mathrm{Li}$ coin cells, the cathode was small disk with a diameter of $0.6 \mathrm{~cm}$. For pouch cells, the cathode size was $4 \mathrm{~cm} \times 5 \mathrm{~cm}$. The multilayer pouch cell contains 16 layers cathodes, and each layer cathode coated with active material on both sides. All the electrochemical performances were tested on Neware CT-4008 battery testing 
system at temperature. 


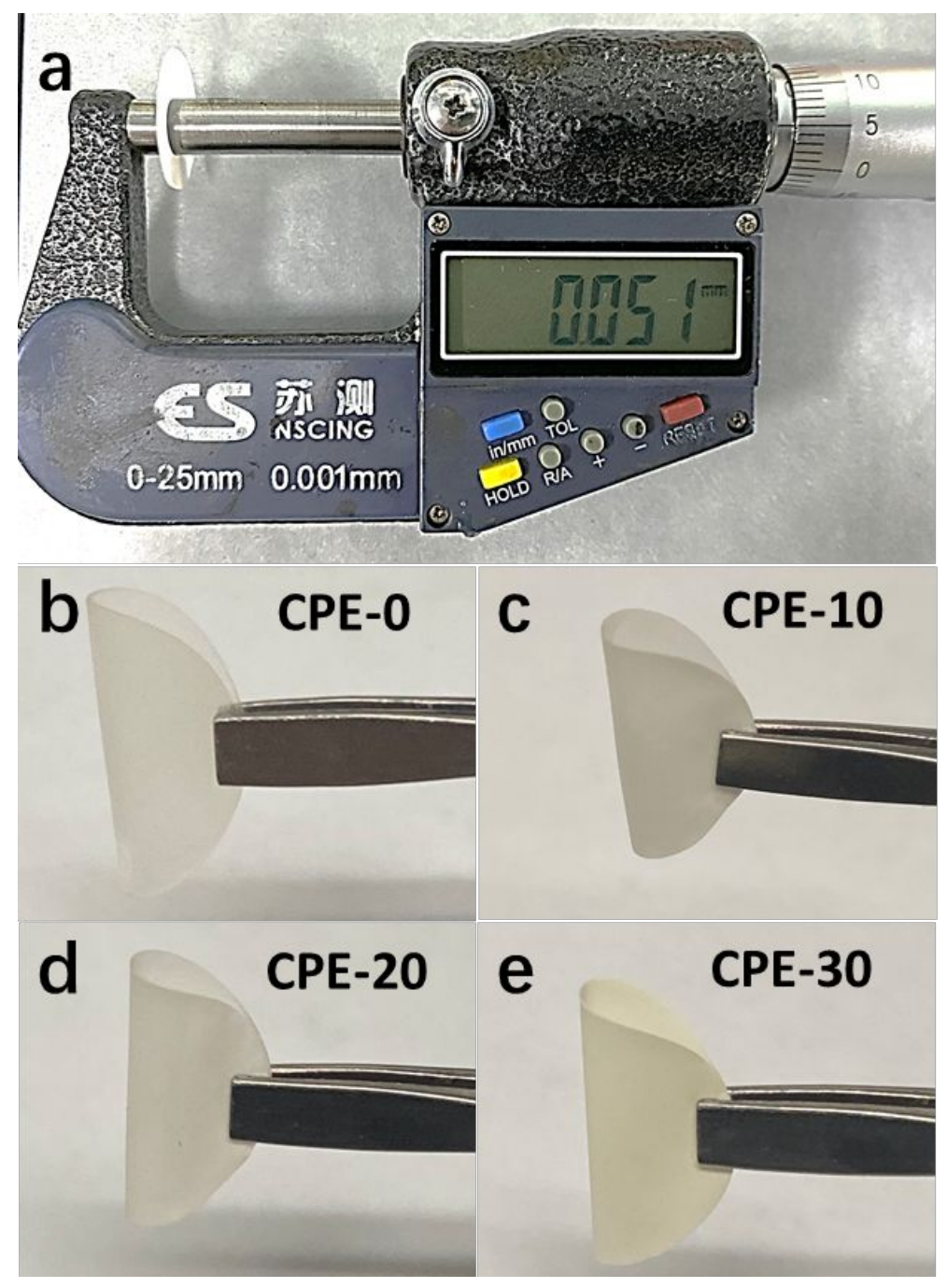

Figure S1. (a) Photo of thickness measurement of CPE membranes; Photos of the (b) CPE-0, (c) CPE-10, (d) CPE-20 and (e) CPE-30 membrane upon $180^{\circ}$ bending.
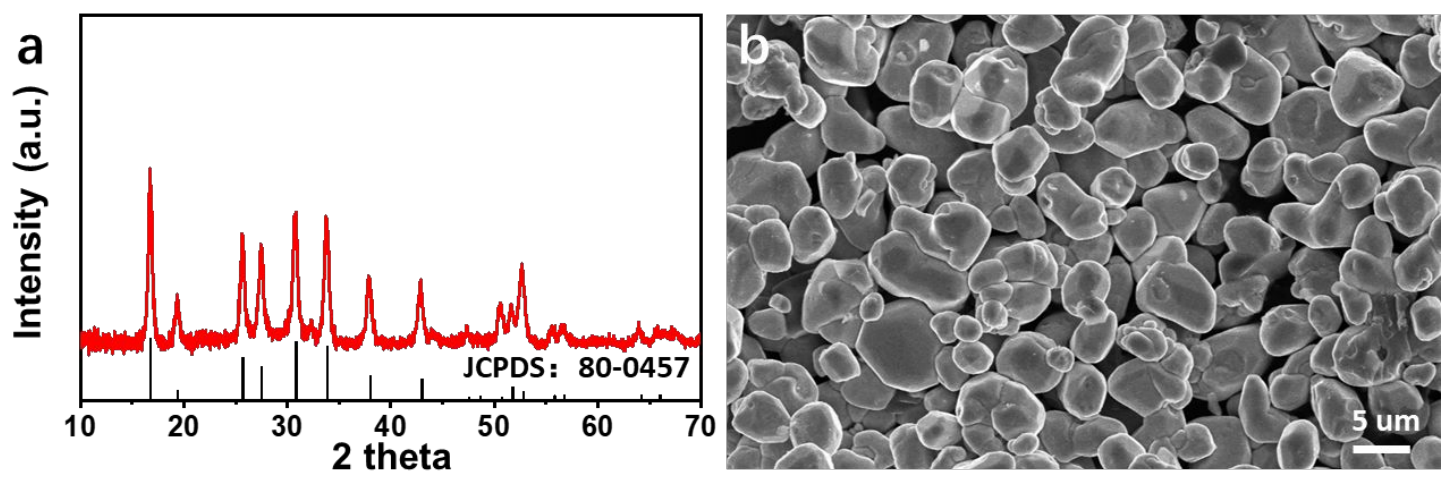

Figure S2. XRD pattern and SEM image of M-LLZTO particles. 


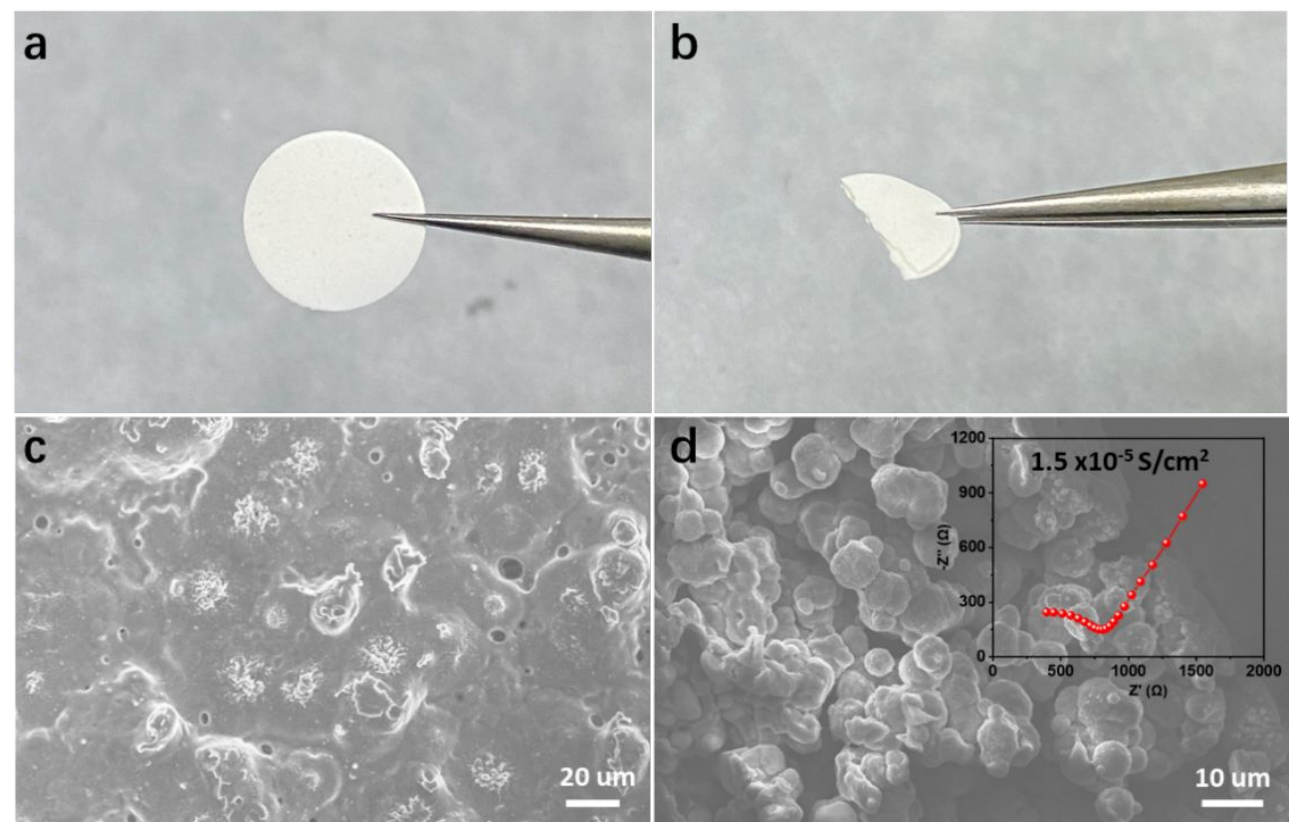

Figure S3. (a,b) Photos of the CPE membrane with 10wt\% M-LLZTO particles; (c) Top-view and (d) cross-sectional SEM images of the CPE membrane with 10wt $\%$ MLLZTO particles.
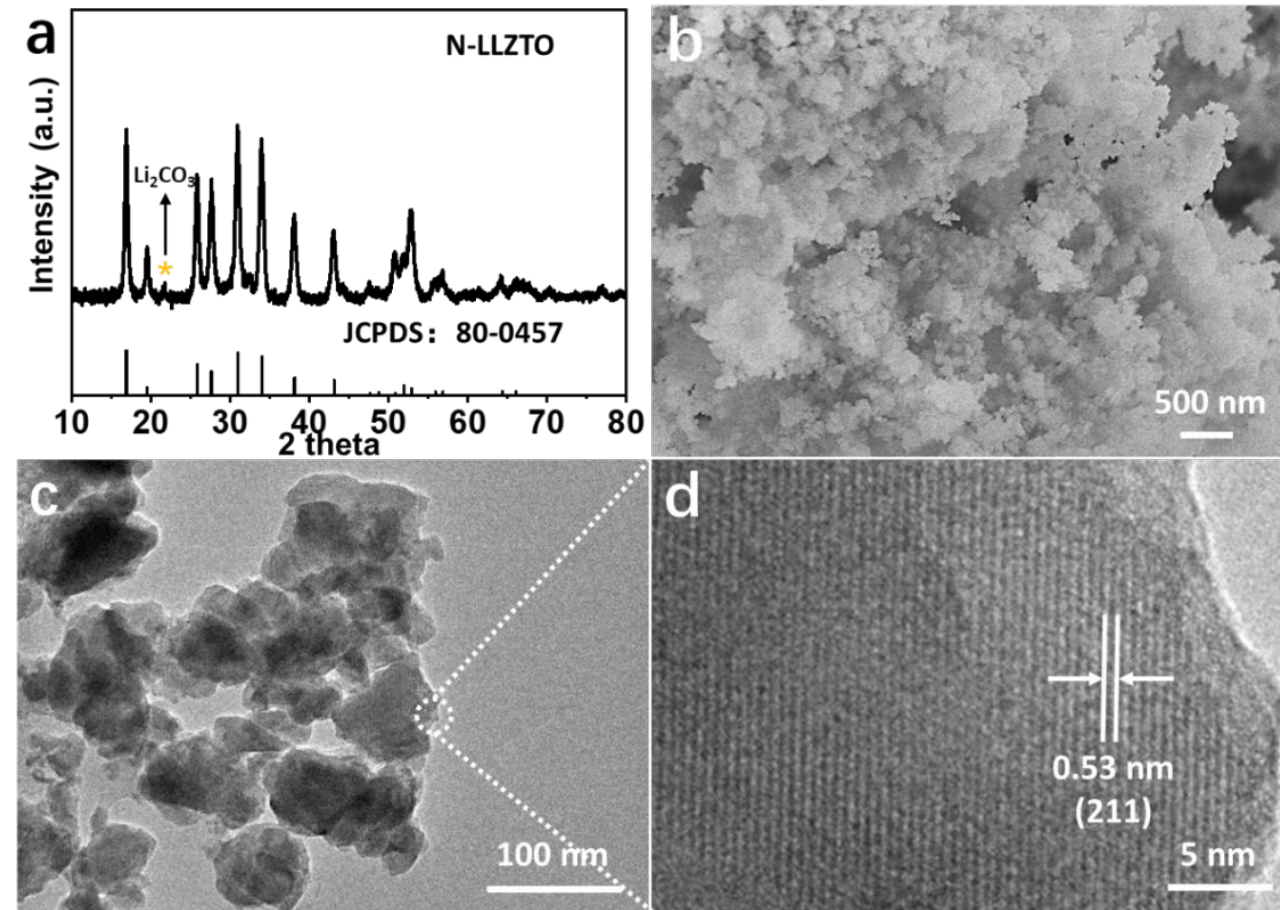

Figure S4. (a) XRD pattern, (b) SEM image, (c) TEM image and (d) high-resolution TEM image of the N-LLZTO particles. 


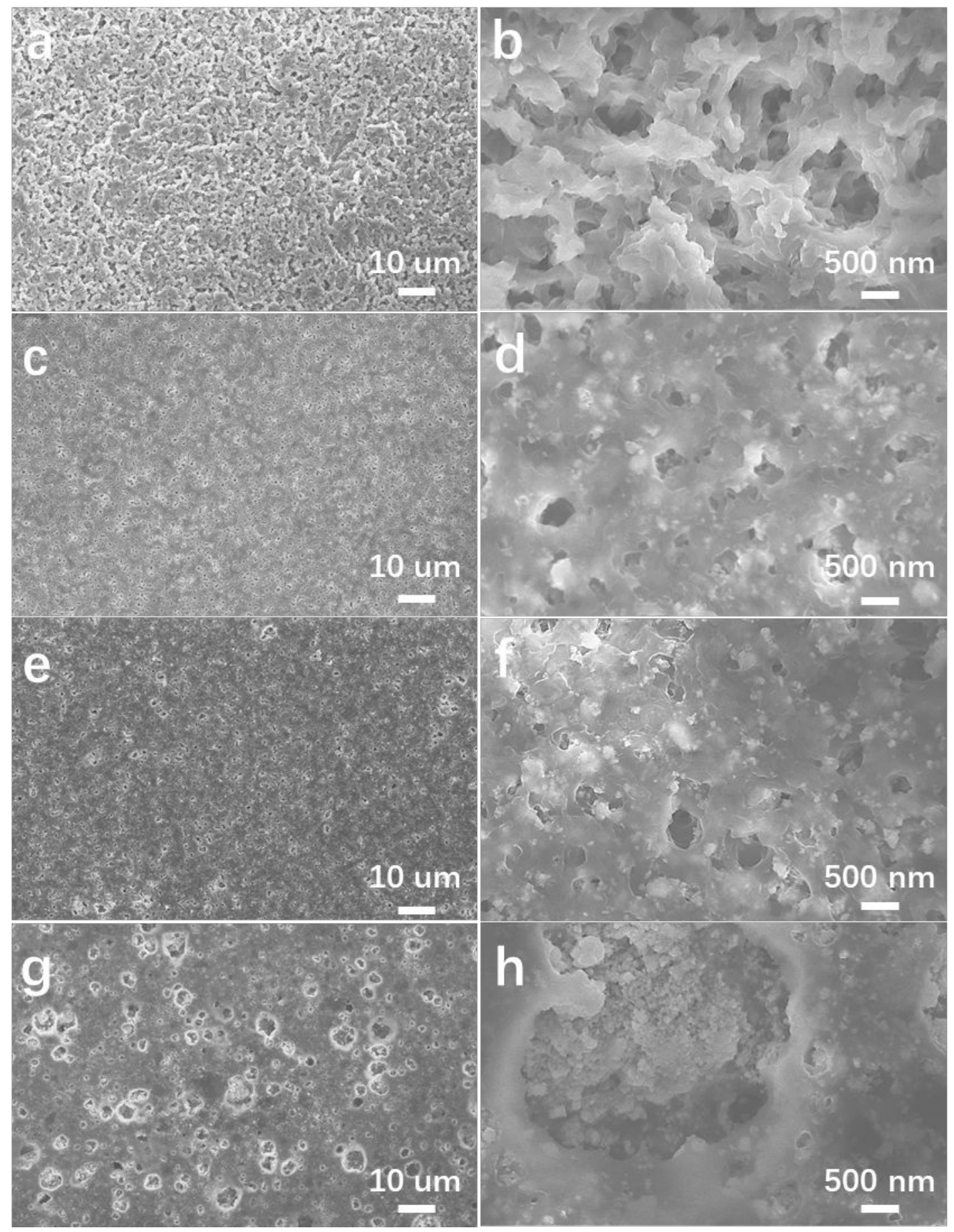

Figure S5. Top-view SEM images of (a,b) CPE-0, (c,d) CPE-10, (e,f) CPE-20, and

(g,h) CPE-30 membranes. 


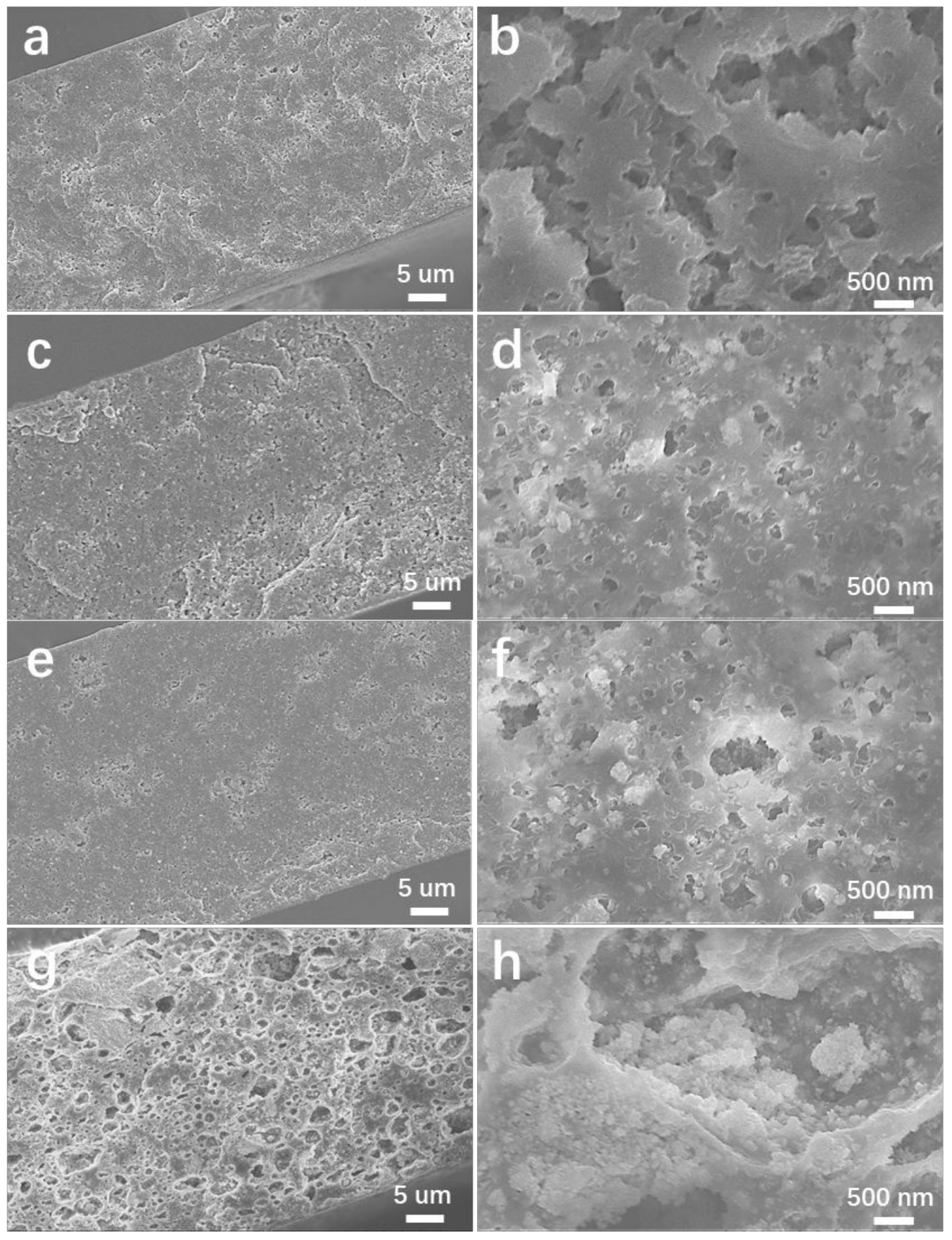

Figure S6. Cross-sectional SEM images of (a,b) CPE-0, (c,d) CPE-10, (e,f) CPE-20, and (g,h) CPE-30 membranes. 


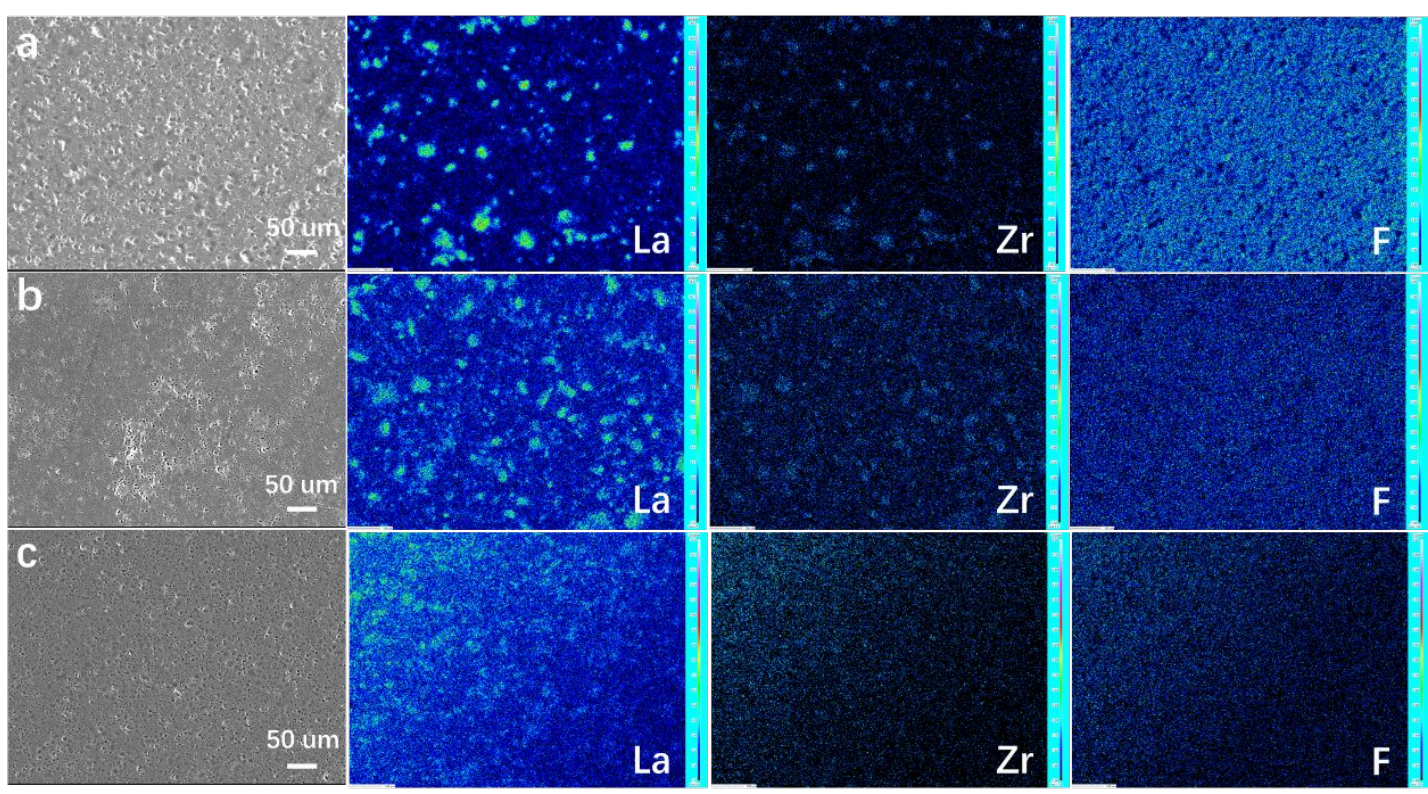

Figure S7. EPMA maps of the (a) CPE-10, (b) CPE-20 and (c) CPE-30 membranes.

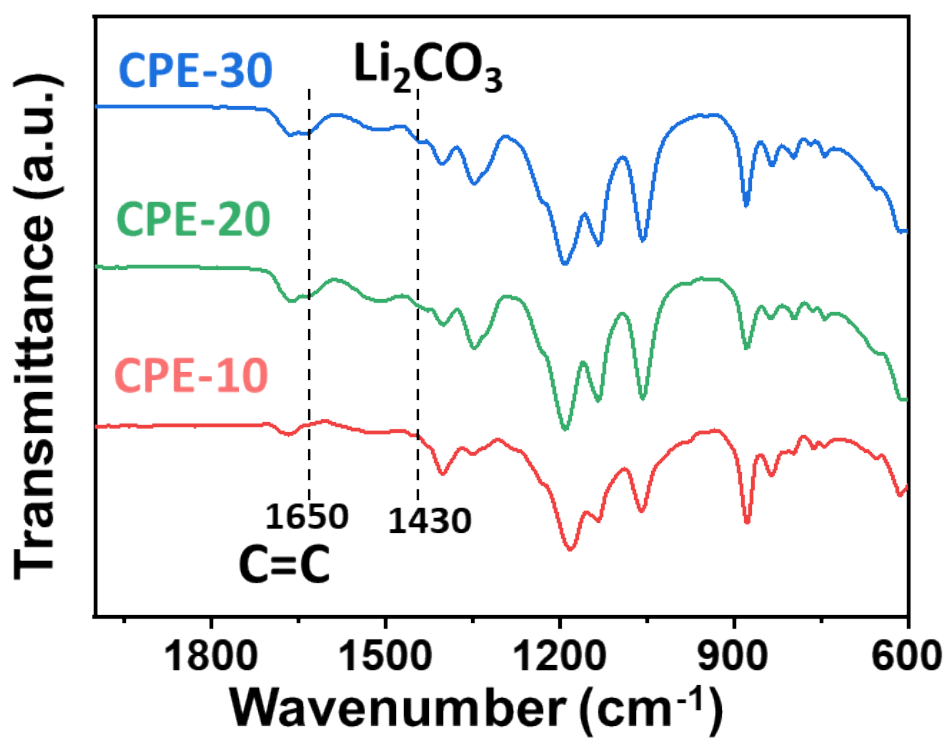

Figure S8. FTIR spectra of the CPE-10, CPE-20 and CPE-30 membranes. 


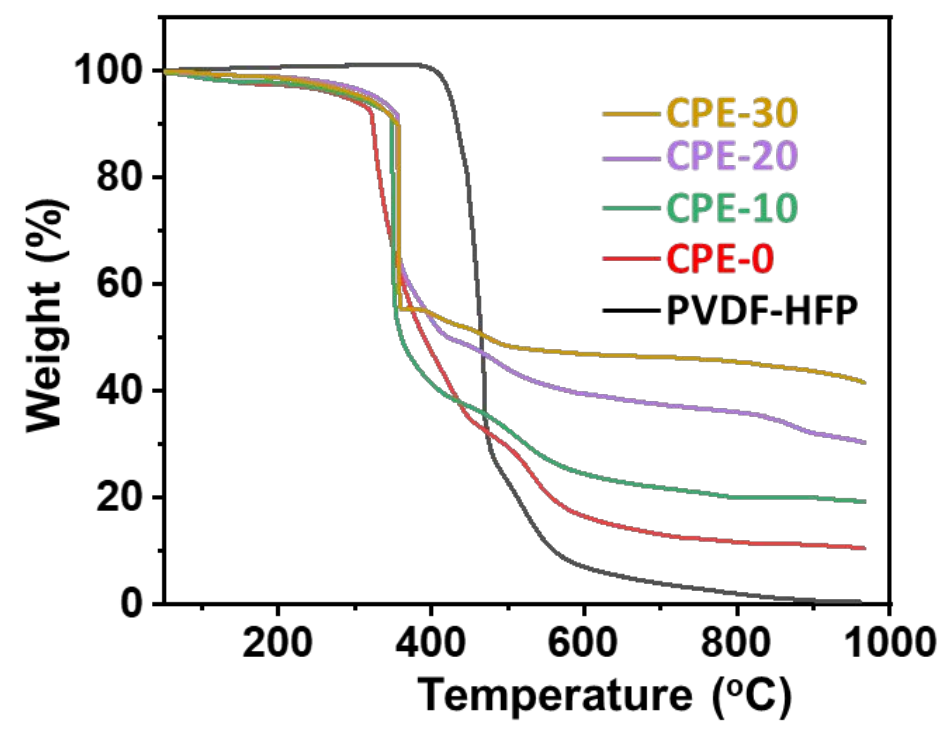

Figure S9. TGA curves of PVDF-HFP, CPE-0, CPE-10, CPE-20 and CPE-30.
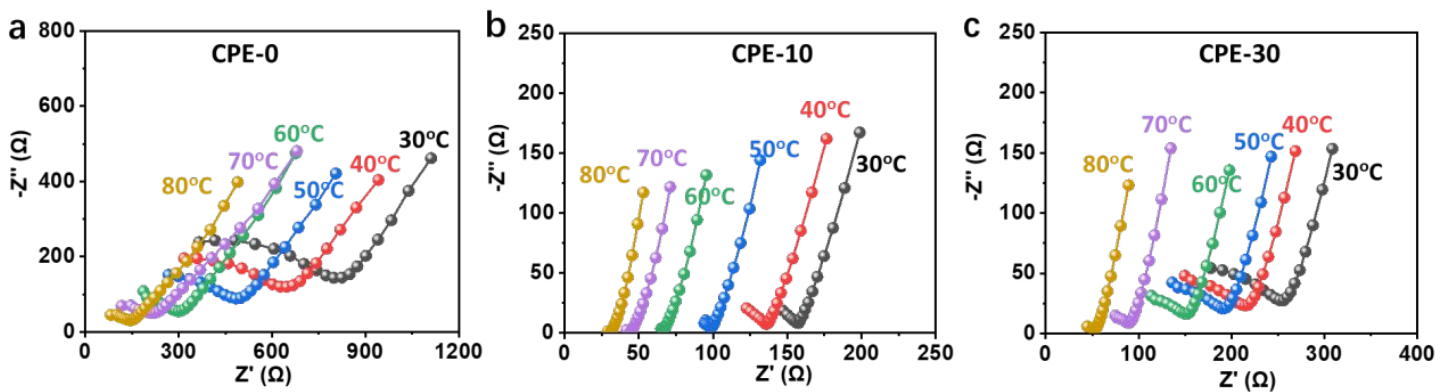

Figure S10. Impedance spectroscopy of (a) CPE-0, (b) CPE-10 and (c) CPE-30 membrane at different temperatures.

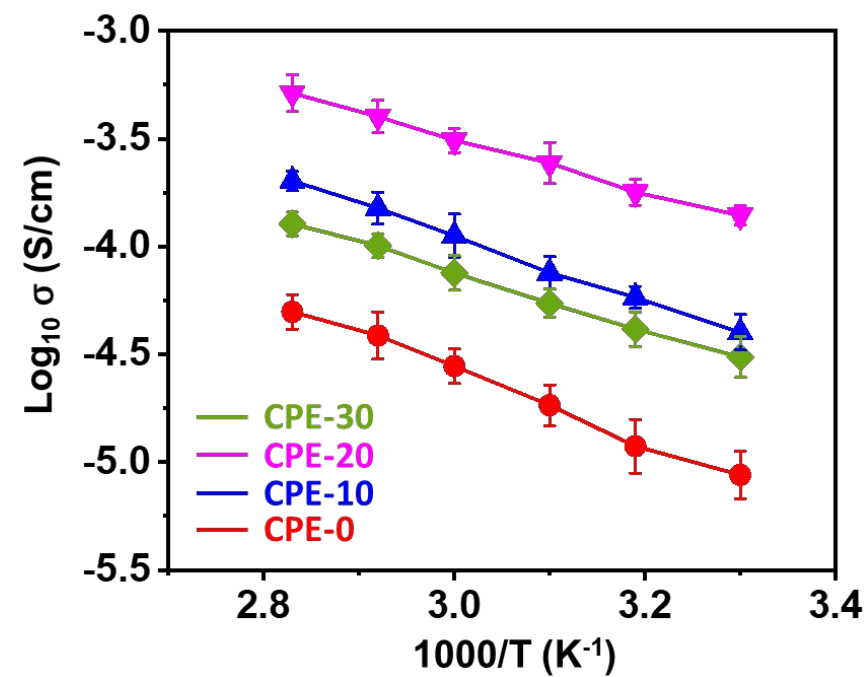

Figure S11. The error bars of Arrhenius plots of CPE-0, CPE-10, CPE-20 and CPE- 
30 membranes.
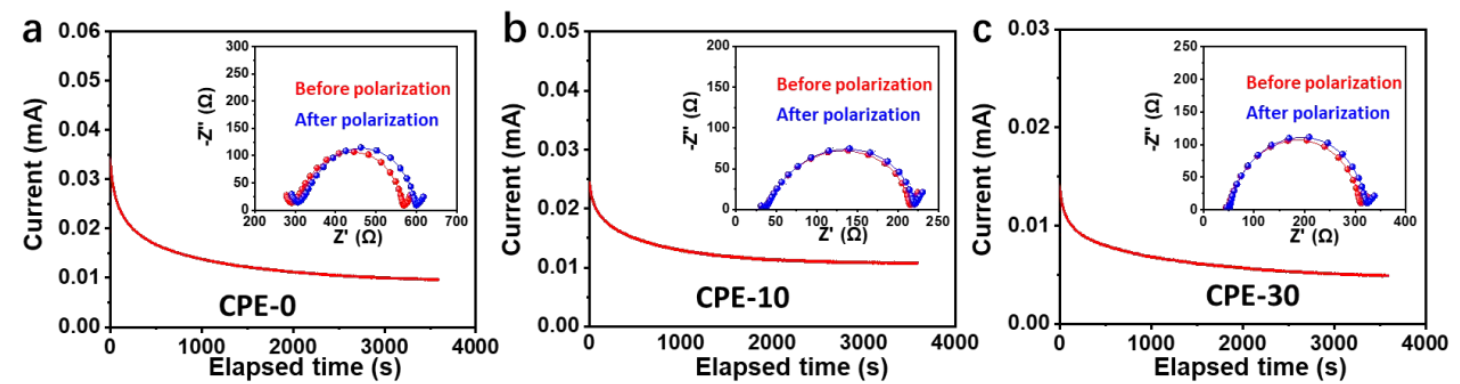

Figure S12. Current time profiles of a symmetrical Li cell with (a) CPE-0, (b) CPE10, and CPE-30. The inset shows the Nyquist impedance spectra before and after polarization.

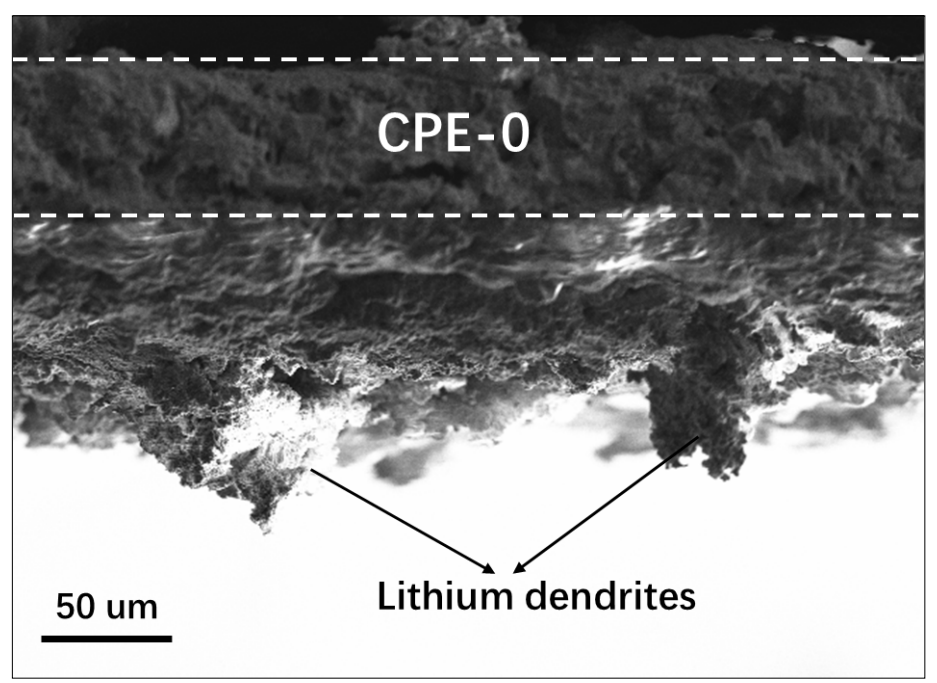

Figure S13. The cross-sectional SEM image of the CPE-0 membrane after long time cycles. 

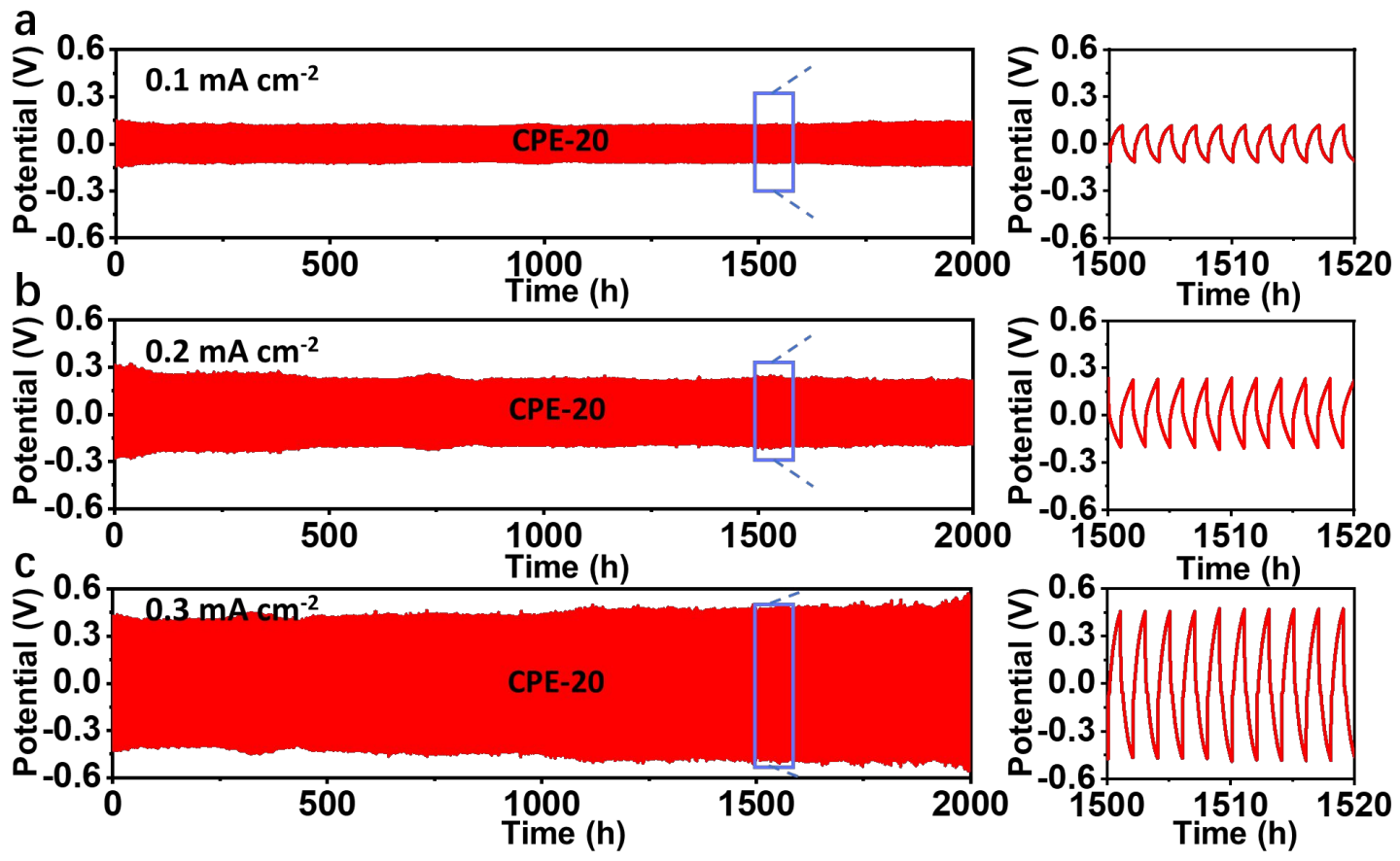

Figure S14. Voltage profiles of Li plating and stripping cycling for the CPE-20 membrane at the current density of (a) 0.5 , (b) 0.2 and (c) $0.3 \mathrm{~mA} \mathrm{~cm}^{-2}$. 


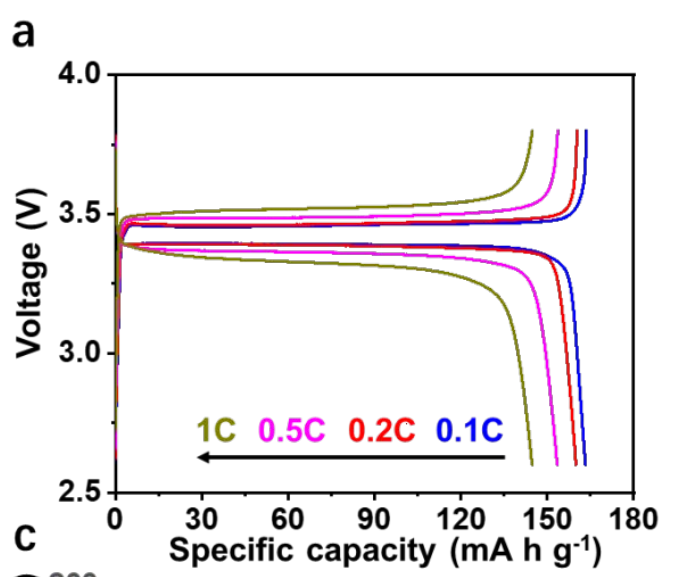

b
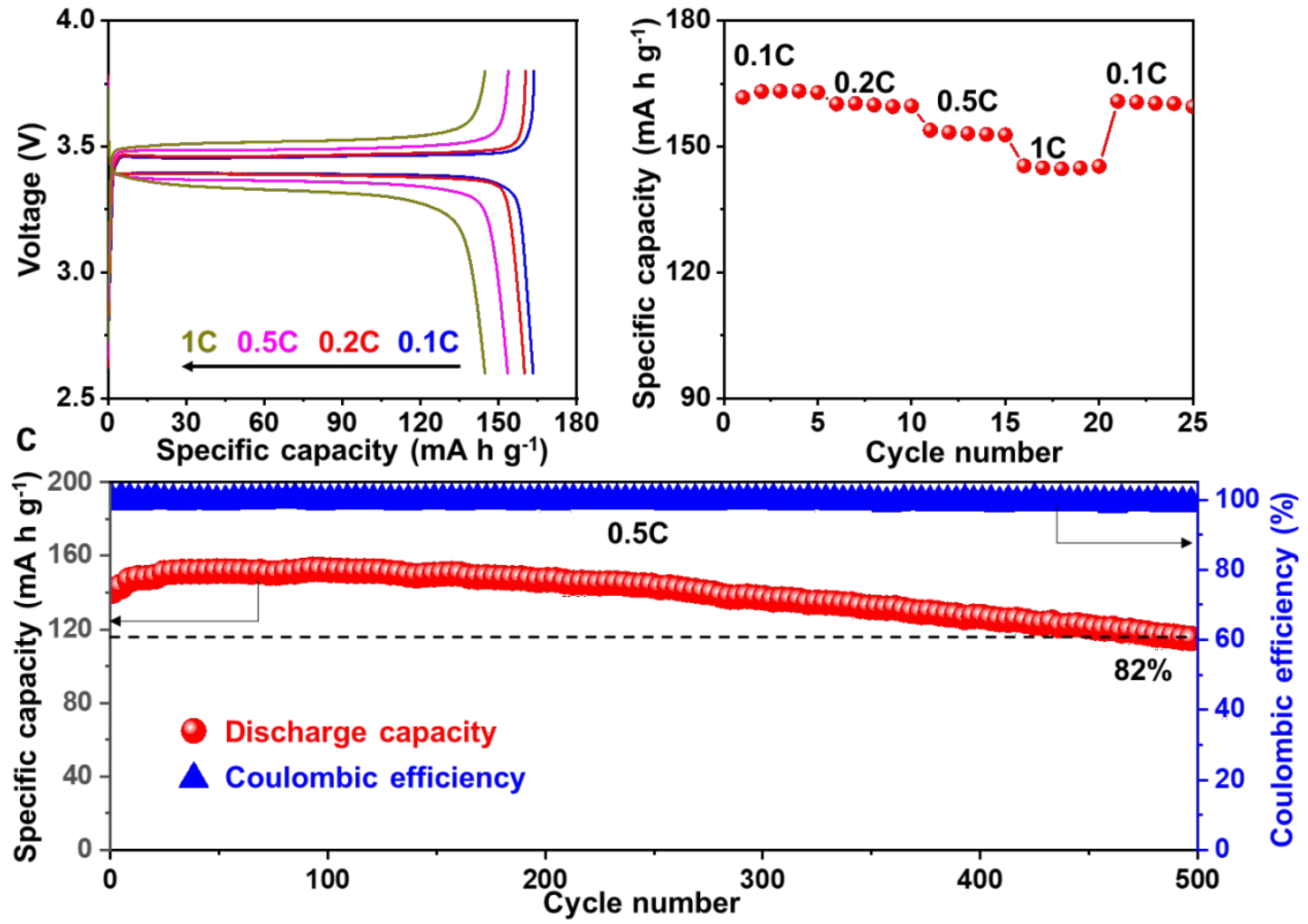

Figure S15. (a) Charge-discharge curves of the $\mathrm{LiFePO}_{4}|\mathrm{CPE}-20| \mathrm{Li}$ coin cell in a voltage range of 2.6-3.8 $\mathrm{V}$ at different current densities and (b) corresponding rate performance; (c) Long-term cycling performance of the $\mathrm{LiFePO}_{4}|\mathrm{CPE}-20| \mathrm{Li}$ cell at $0.5 \mathrm{C}$. 